\section{Paraneoplastic dermatomyosi- tis and prostate cancer: Myopathy regression under cancer-directed therapy}

\author{
Mafalda Miranda Baleiras, \\ Luís Maduro, Carolina Vasques, \\ Filipa Ferreira, Marta Mesquita Pinto, \\ Ana Martins \\ Hospital São Francisco Xavier, Centro \\ Hospitalar Lisboa Ocidental, Portugal
}

\begin{abstract}
Prostate cancer is the second most frequent malignancy in men worldwide and the fifth leading cause of death. Dermatomyositis (DM) is a rare idiopathic inflammatory myopathy characterized by musculocutaneous manifestations. However, DM can also present as a paraneoplastic syndrome of an underlying neoplasm. We report a case of a 65-year-old man diagnosed with prostate adenocarcinoma in the setting of severe dysphagia, muscle weakness and a facial erythematous rash. At first, the DM-related symptoms resolved with the initial treatment for the underlying malignancy. Yet, they flared up as the tumor progressed. To sum up, DM is a rare systemic disorder with unknown etiology. There is a well-established association between DM and malignancy. Malignancy-headed therapy can improve DM manifestations and the recurrence of DM symptoms may act as an early warning of malignancy relapse.
\end{abstract}

\section{Introduction}

Paraneoplastic syndrome (PS) is a rare entity, occurring in up to $8 \%$ of cancer patients. ${ }^{1}$ Dermatomyositis (DM) is an uncommon idiopathic inflammatory myopathy (IIM) characterized by muscle inflammation and weakness, well-defined cutaneous lesions and systemic complications. The extension of muscle and cutaneous manifestations varies. The reported annual incidence of adult DM ranges from 0.5 to 0.89 per 100,000 , affecting mostly middle-aged women. The female to male ratio is $2: 1{ }^{2}$ The majority of cases is idiopathic, but in $15-30 \%$ of adult-onset DM cases an underlying malignancy precipitates the PS manifested as DM. ${ }^{3}$ Patients with simultaneous DM and cancer have a poor prognosis. ${ }^{4}$ Hence, early recognition of this disease is important.

Herein, our rare case of concurrent DM and prostate cancer underlines two remarkable relationships: DM symptoms improve after cancer-specific therapy and work as an early warning of neoplasm progression. The case makes also clear how the diagnosis of $\mathrm{DM}$ in an adult should raise suspicion of an underlying malignancy; this may help to secure timely and safe patient care. This result is particularly impactful as the case concerns the second largest worldwide male malignacy, prostate cancer. ${ }^{5}$

\section{Case Report}

A 65-year-old Cape Verdean, with a past medical history of type 2 diabetes and hypertension, presented with a five-month history of progressive asthenia, dysphagia and tetraparesis. He reported no other systemic symptoms and denied a family history of malignancy.

On examination, the subject showed a fixed painless $2 \mathrm{~cm}$-long left supraclavicular lymph node and a mild periorbital violaceous erythema. Neurological examination revealed reduced symmetrical proximal strength in both upper and lower limbs $(3 / 5)$. He was unable to rise independently from a seated position and displayed a $\mathrm{wad}$ dling gait. General examination was unremarkable.

A clinical diagnosis of DM was made, supported on raised creatine kinase (CK) of $1450 \mathrm{U} / \mathrm{L}$ (reference range $<190$ ) and lactate dehydrogenase (LDH) of 580 U/L (reference range 135-225). Myositis-specific antibody panel was positive for the antinuclear matrix protein 2 (NXP2), thus corroborating this diagnosis. On further investigation, a computed tomography (CT) scan of the chest, abdomen and pelvis revealed a nodular prostate and enlarged left iliac, para-aortic and supraclavicular lymph nodes (Figure 1). A transperineal prostate biopsy showed a Gleason grade group 5 acinar adenocarcinoma. Therefore, the patient was diagnosed with very high-risk metastatic hormone-sensitive prostate cancer with paraneoplastic dermatomyositis.

Following DM diagnosis, physical rehabilitation, and a five-day course of 2 $\mathrm{g} / \mathrm{kg} \quad$ bodyweight Intravenous Immunoglobulin (IVIG) was prescribed as the initial therapy. Albeit with some improvement of the skin lesions and preadmission muscle-associated enzyme levels, the muscle weakness persisted, and he developed the inability to dress himself and perform daily hygiene.

Later, the neoplasm was approached with a combination of abiraterone ( $1 \mathrm{~g} /$ day) with prednisolone $(10 \mathrm{mg} /$ day $)$ and andro-
Correspondence: Mafalda Miranda Baleiras, Hospital São Francisco Xavier, Centro Hospitalar Lisboa Ocidental, Estr. Forte do Alto Duque, 1449-005 Lisboa.

Tel.: +351913147616

E-mail: ambaleiras@gmail.com

Key words: Prostate Cancer, Paraneoplastic Syndrome, Dermatomyositis, MyositisSpecific Antibody, Recurrence.

Acknowledgments: We acknowledge the important contribution of the patient willingness to collaborate through the conduction of his case.

Contributions: MMB: literature review and writing of the original manuscript. LM: editing. CV: supervision and resources. FF: writing-review and editing, supervision, and resources. MMP: supervision and resources. AM: supervision and resources. All authors have read and agreed to the published version of the manuscript.

Conflict of interest: The authors declare no potential conflict of interests.

Funding: None.

Ethical approval and informed consent: Informed consent was obtained from the patient.

Availability of data and material: Data and materials are available from the authors upon request.

Please cite this article as: Baleiras MM, Maduro L, Vasques C, et al. Paraneoplastic dermatomyositis and prostate cancer: Myopathy regression under cancer-directed therapy. Dermatol Rep 2021;13:9262.

Received for publication: 11 May 2021.

Accepted for publication: 29 July 2021.

This work is licensed under a Creative Commons Attribution-NonCommercial 4.0 International License (CC BY-NC 4.0).

${ }^{\circ}$ Copyright: the Author(s), 2021

Licensee PAGEPress, Italy

Dermatology Reports 2021; 13:9262

doi:10.4081/dr.2021.9262

gen deprivation therapy made up of leuprorelin (22.5 mg every 12 weeks) and bicalutamide (50 mg/day). His weakness and rash resolved and CK levels normalized as the prostate-specific antigen (PSA) started to decrease progressively from 77.6 $\mathrm{ng} / \mathrm{ml}$ to $14.8 \mathrm{ng} / \mathrm{mL}$. He finally managed to eat solid consistent food and became fully independent in his daily life activities.

Eight months after the prostate cancer- 
directed therapy had begun, blood tests showed elevation of PSA levels (to 40 $\mathrm{ng} / \mathrm{mL}$ ). At the same time, the patient complained again of muscle weakness in the limbs - yet, the neurologic exam revealed no deficit of muscle strength. One month later, a recurrence of the DM clearly emerged including proximal weakness, dysphagia, diffused myalgia and raised CK (439 U/L). A CT scan was conducted and revealed ganglionar progression (Figure 2). The patient was then administered with docetaxel-based ( $75 \mathrm{mg} / \mathrm{m}^{2}$ every 21 days) systemic chemotherapy. After the first cycle of chemotherapy, his muscle claims decreased remarkably. The authors keep monitoring this case closely.

\section{Discussion}

DM typically presents with characteristic skin lesions and progressive symmetrical proximal muscle weakness. Myopathy is present in approximately $80 \%$ of patients with DM and is usually painless. Dysphagia, dysphonia and aspiration pneumonia may also occur and are associated with a poor prognosis. In addition to pathognomonic cutaneous findings, such as Gottron's papules and heliotrope rash, DM often presents with periungual telangiectasias, poikiloderma and cuticular overgrowth. ${ }^{6}$ Lesions can precede or follow myositis and may be pruritic, which normally disturbs life quality. ${ }^{7}$

The European League Against Rheumatism and the American College of Rheumatology (EULAR/ACR) criteria for IIM, published in 2017, are a highly sensitive and specific probability-based classification system. A classification tree is then used to assign IIM subtypes. ${ }^{8}$ Although its pathogenesis remains unclear, several genetic, immunologic and environmental factors are thought to play a role in DM development. ${ }^{9}$ Approximately $70 \%$ of DM patients have myositis-specific autoantibodies (MSA) in circulation. Their presence matters for prognosis and clinical management inasmuch as they are associated with distinct clinical phenotypes. ${ }^{10}$

Paraneoplastic dermatomyositis was first described in 1916 and accounts for approximately $30 \%$ of dermatomyositis cases. ${ }^{11}$ The risk of malignancy is highest within a year of DM diagnosis and remains high for up to five years. A neoplasm can precede, coincide with or follow the diagnosis of DM. ${ }^{12}$ Although the precise link between malignancy and DM is poorly understood, cellular and humoral immunologic abnormalities have been suggested. The most frequent associated malignancies are breast, ovarian, lung, colorectal and Hodgkin's lymphoma. Malignancy-associated DM risk factors are: age over 60, male gender, dysphagia, skin necrosis, cutaneous vasculitis, rapid onset $(<$ weeks), elevated $\mathrm{CK}$ and C-reactive protein and an increase in the erythrocyte sedimentation rate (ESR). The association between DM and malignancy is particularly strong in those patients with antibodies to transcription intermediary factor $1 \gamma(\mathrm{TIF} 1 \gamma)$ or nuclear matrix protein 2 (NXP2), some of the MSA recently discovered. ${ }^{13}$

DM treatment goals are the improvement of daily living activities through increased muscle strength and the alleviation of extramuscular manifestations. Systemic corticosteroids are the mainstay of therapy. Second-line therapeutic regimens include methotrexate and azathioprine. If resistance to therapy is developed, rituximab or intravenous immunoglobulin can be used ${ }^{14}$. Supplemental nutrition and rehabilitation are also essential to improve mus-
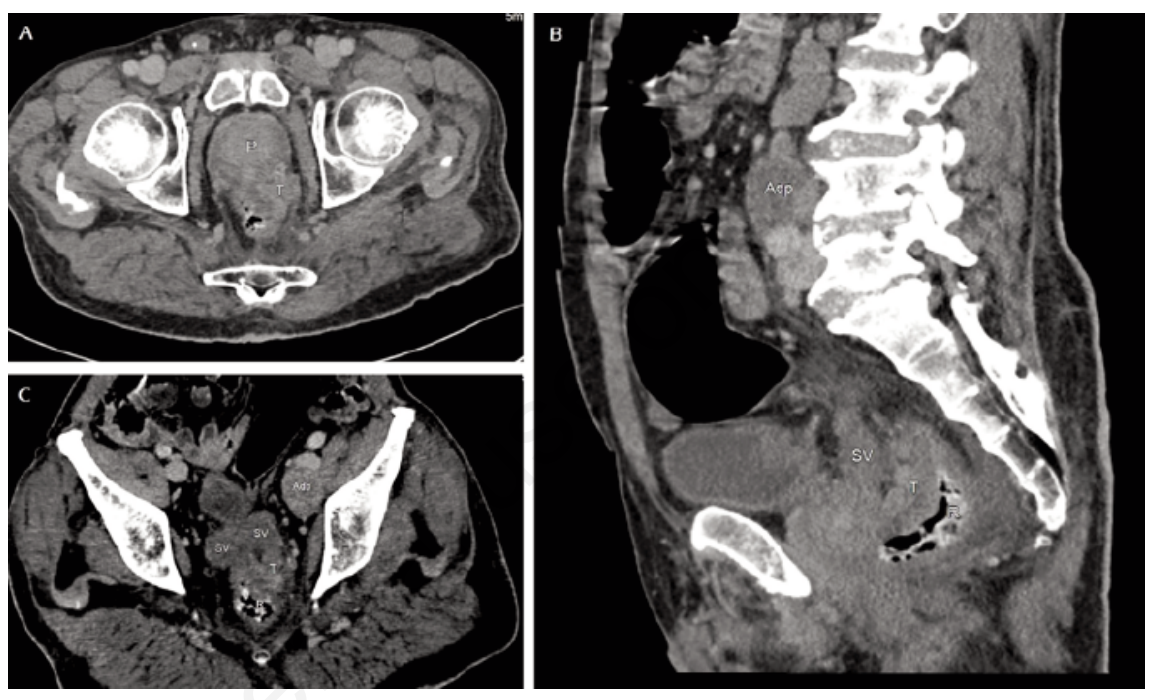

Figure 1. Image A: Oblique axial CT plane shows an enlarged and asymmetric prostate (P) with prominent nodular densities extending posteriorly into the periprostatic space. Transrectal prostate biopsy revealed cancer in this area. Image B: Sagittal CT plane. Image C: different oblique axial CT plane. These latter planes demonstrate the tumor (T) also spreading superiorly, with infiltration of fat between the left seminal vesicle (SV) and the rectum wall $(\mathrm{R})$ and loss of the fat planes between these structures. CT is not accurate to characterize locally the prostatic cancer. MRI is the imaging method for regional staging, but the presence of disseminated disease, shown by non-regional/distant metastatic adenopathies (Adp), made MRI unnecessary.

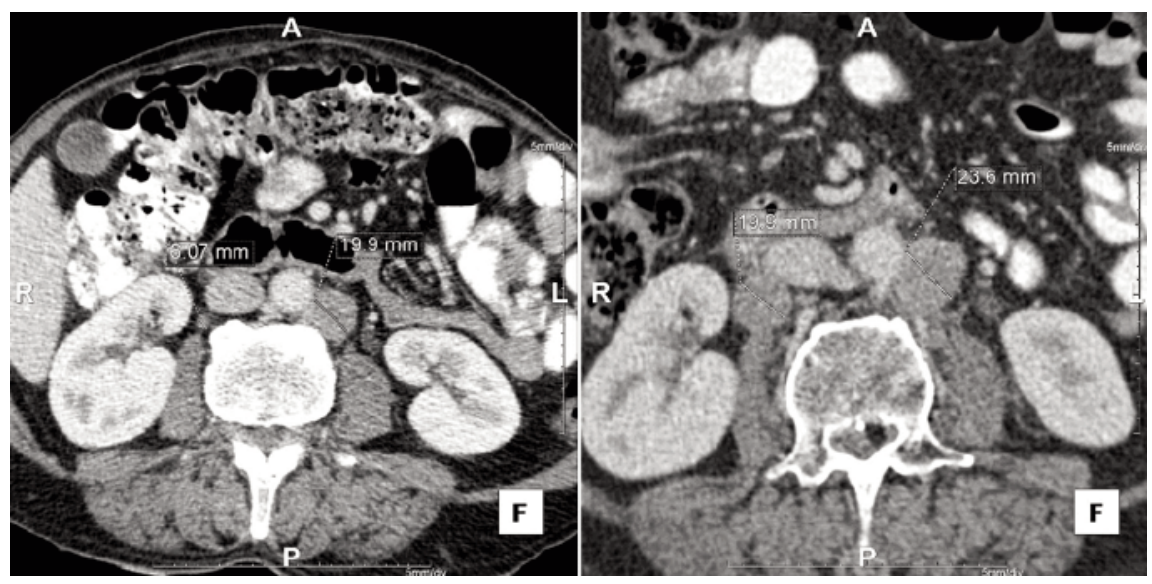

Figure 2. CT images. The right image (Axial CT scan) shows progressive disease with an increase of retroperitoneal lymph nodes, comparing to the CT performed nine months before (left image). 
cle strength and endurance. Some studies report improvement of DM symptoms with cancer treatment and the relation of myopathy recurrence with neoplasm relapse, thus supporting a paraneoplastic nature. ${ }^{15}$ There are also reports highlitnening DM symptom resolution after treatment for the underlying malignancy, thereby avoiding the use of corticotherapy and its side effects. ${ }^{16,17}$

In the current case, the clinical suspicion for DM arose from typical features such as scapular and pelvic girdles muscle weakness, erythematous skin rash and elevated CK. The anti-NXP2 positivity also supported the diagnosis, since this DM-specific autoantibody is present in up to $30 \%$ of adult-onset DM. Anti-NXP2 antibodies are associated with heliotrope rash and more extensive myopathy including dysphagia, as observed in our patient. Fiorentino et al. reported that $24 \%$ of anti-NXP2 positive DM patients had malignancy. This association with cancer is also supported in a Japanese cohort study showing that three out of eight anti-NXP2 positive patients had advanced stage malignancy. ${ }^{18}$ The patient hereby considered also met other risk factors for cancer such as male gender, ageing, dysphagia and diabetes. There were two developments in his condition in line with the paraneoplastic origin of DM: i) the significant clinical enhancement with the cancer-directed therapy; and ii) the DM recurrence coincided with the tumor progression.

\section{Conclusions}

To sum up, DM is a rare systemic disorder with unknown etiology. There is a wellestablished association between DM and malignancy. Thus, the chance of an underlying neoplasm should be ruled out in newly diagnosed DM especially in high-risk patients. A coordinated multidisciplinary approach is essential for early diagnosis and timely treatment to improve the malignan- cy-associated DM prognosis.

The treatment of the underlying malignancy will usually result in symptom resolution in cases of paraneoplastic DM. It is important to keep in mind that myositis manifestations may relapse in the setting of recurrent or aggravated malignancy. These patients should therefore be monitored closely.

\section{References}

1. Baijens LW, Manni JJ. Paraneoplastic syndromes in patients with primary malignancies of the head and neck. Four cases and a review of the literature. Eur Arch Otorhinolaryngol 2006;263:32-6.

2. Stockton D, Doherty VR, Brewster DH. Risk of cancer in patients with dermatomyositis or polymyositis, and follow-up implications: a Scottish populationbased cohort study. Br J Cancer 2001; 85:41-5.

3. Olazagasti JM, Baez PJ, Wetter DA, Ernste FC. Cancer risk in dermatomyositis: a meta-analysis of cohort studies. Am J Clin Dermatol 2015;16: 89-98.

4. Motomura K, Yamashita H, Yamada S, et al. Clinical characteristics and prognosis of polymyositis and dermatomyositis associated with malignancy: a 25-year retrospective study. Rheumatol Int 2019;39:1733-9.

5. Rawla P. Epidemiology of Prostate Cancer. World J Oncol 2019;10:63-8.

6. Schmidt J. Current Classification and Management of Inflammatory Myopathies. J Neuromuscul Dis 2018;5:109-29.

7. Dalakas MC. Inflammatory muscle diseases. N Engl J Med 2015;372:1734-47.

8. Bottai M, Tjärnlund A, Santoni G, et al. EULAR/ACR classification criteria for adult and juvenile idiopathic inflamma- tory myopathies and their major subgroups: a methodology report. RMD Open 2017;3:e000507.

9. DeWane ME, Waldman R, Lu J. Dermatomyositis: Clinical Features and Pathogenesis. J Am Acad Dermatol 2020;82:267-81.

10. Calvão J, Azeiteiro ARI, Gonçalo M. A importância dos novos autoanticorpos específicos da dermatomiosite. J Portug Soc Dermatol Venereol 2019;77:15-24.

11. Hill CL, Zhang Y, Sigurgeirsson B, et al. Frequency of specific cancer types in dermatomyositis and polymyositis: a population-based study. Lancet 2001;357:96-100.

12. Kang E, Lee S, Ascherman D, et al. Temporal relationship between cancer and myositis identifies two distinctive subgroups of cancers: impact on cancer risk and survival in patients with myositis. Rheumatology 2016;55:1631-41.

13. Jakubaszek M, Kwiatkowska B, Maślińska M. Polymyositis and dermatomyositis as a risk of developing cancer. Reumatologia 2015:53:101-5.

14. Waldman R, DeWane ME, Lu J. Dermatomyositis: Diagnosis and Treatment. J Am Acad Dermatol 2020;82:283-96.

15. Castro AS, Barroso A, Parente B. Dermatomiosite como primeira manifestação de uma neoplasia pulmonar. Rev Port Pneumol 2013;19:179-83.

16. Lin C, Ying Z, Sijing C. Spontaneous resolution of dermatomyositis associated with fallopian-tube carcinoma following staging surgery: A case report. Medicine 2019;98:e14530.

17. Orth T, Galle PR, Mayet WJ. Severe dermatomyositis associated with lymph node recurrence of an endometrial carcinoma. J Clin Rheumatol 1999;5:41-2.

18. Nakashima R. Clinical significance of myositis-specific autoantibodies. Immunol Med 2018;41:3,103-12. 